\title{
Advances in cancer genetics
}

\author{
Shirley Hodgson
}

At the time that I entered the field of clinical genetics in the early 1980s there was an explosion of gene identification leading to a rapid improvement in our ability to diagnose genetic disease and to offer screening and prenatal diagnosis. The major emphasis was on advising and counselling individuals with genetic disorders, or families with children affected with disorders which could have a genetic component. The geneticist would make a genetic diagnosis, and advise those affected with such disorders about its cause and natural history and the chance of recurrence of the condition in the family. Prenatal diagnosis was available for some conditions, mainly by ultrasound scanning, and amniocentesis for chromosomal and biochemical anomalies. With the discovery of the genes involved in the causation of many disorders, it become possible to offer accurate genetic testing, predictive tests and, where appropriate, prenatal diagnosis to couples with affected children, based on the detection of the underlying mutation.

When I become a consultant in clinical genetics at Addenbrooke's Hospital, Cambridge, in 1988 cancer genetics was not a well-established part of the clinical genetics service, and there were few referrals for a discussion of cancer risks in relation to genetic cancer susceptibility apart from single gene disorders such as familial adenomatous polyposis (FAP), where the gene penetrance was high, the phenotype obvious and the inheritance clear. Only a few centres offered a specific cancer genetics service for individuals with a family history of colorectal or breast cancer, notably the pioneering Family Cancer Clinic at St Mark's Hospital, London, run by Joan Slack with Victoria Murday, the cancer genetics clinics at St Mary's Hospital, Manchester (Gareth Evans and Tony Howell started this clinic in 1988), and some clinics at Guy's Hospital, London, run by Bruce Ponder. Joan Slack saw patients who had a family history of colorectal cancer (CRC), and advised them about their level of risk based on family history. These relative risks were derived from the study by Lovett, ${ }^{1}$ from the incidence of CRC in the close relatives of patients attending St Mark's, analysed by Richard Houlston. ${ }^{2}$ Individuals at low risk were reassured, those at moderate risk were offered five-yearly colonoscopies from middle age (three-yearly if polyps were detected), and high-risk families were evaluated further to see if they had evidence of hereditary non-polyposis colorectal cancer (HNPCC, Lynch syndrome), FAP or another high-risk cancer susceptibility syndrome. ${ }^{3}$ The recognition and management of families with HNPCC had been pioneered by Henry Lynch with his son, Patrick, who recognised that it was an important hereditary cause of CRC susceptibility which required colonoscopic surveillance of affected individuals and their at-risk relatives. ${ }^{4}$

I became interested in cancer genetics in 1988 and, with Eamonn Maher (then a registrar in clinical genetics at Addenbrooke's), wrote the first handbook of cancer genetics aimed at the non-specialist clinician. I returned to Guy's Hospital in 1990 and received generous support from Sir Walter Bodmer and the Imperial Cancer Research Fund to enable me to work in the St Mark's Familial Cancer Clinics after Joan Slack retired. There was a separate clinic at St Mark's, the Polyposis Registry, which cared for families with FAP. The registry was initially established in 1925, with the publication of the clinical details of three families with FAP, ${ }^{5}$ and, with its development into the St Mark's Hospital Polyposis Registry between 1985 and 1986, it became a pioneering paradigm for the management of families with FAP, overseeing the care of affected individuals, and responsible for the ascertainment and screening of at-risk individuals. ${ }^{6}$ Evidence-based management guidelines were developed for patients, and it could clearly be shown that at-risk individuals under surveillance by regular sigmoidoscopy or colonoscopy and subsequent prophylactic surgery for affected individuals, had significantly reduced morbidity and mortality from CRC. The registry also provided the clinical resource for the study of manifestations of the disease, and participated in international collaborative research into the disorder as part of a worldwide network of polyposis registries. They ran clinics where patients were reviewed regularly, and at-risk relatives were seen and screened for colorectal adenomas. They maintained responsibility for these families and developed a relationship with them, which encouraged compliance with screening.

No genetic tests were available for these high penetrance disorders at the time, so individuals whose parents were affected with FAP or HNPCC were advised to have surveillance, since they were at 50\% risk of inheriting the disorder. For FAP this meant annual sigmoidoscopies or colonoscopies from the early teens, and for HNPCC, colonoscopies every
Shirley Hodgson DM FRCP, Professor of Cancer Genetics, St Georges, University of London 
one-to-two years from 25 years of age. In 50\% of such individuals therefore, this surveillance was unnecessary. It was only in 1991 with the discovery of the adenomatous polyposis coli (APC) gene, ${ }^{7}$ and later that decade the genetic basis of HNPCC, that our understanding of the pathogenesis of these inherited cancer susceptibility syndromes was revolutionised, with the potential for identifying at-risk individuals by genetic testing and offering appropriate cancer surveillance based on an accurate molecular genetic diagnosis. Prenatal diagnosis was also theoretically possible, but the take-up rate was low. It soon became apparent that there were also strong genotype-phenotype correlations, such that certain APC mutations were associated with particularly severe disease, and others with mild, or even 'attenuated' polyposis, and some mutations were more likely to cause the extracolonic features of the disease, like the black fundal pigmentation known as congenital hypertrophy of the retinal pigment epithelium (CHRPE), desmoid disease or the cribriform variant of papillary thyroid cancer. These observations also allowed some deductions to be made about the functions of different regions of the APC gene, and to mutation-related clinical management. The APC gene functions as a tumour suppressor in the main, which conformed to the Knudsen model of inherited cancer susceptibility due to an inherited defect in a tumour suppressor gene facilitating cancer initiation by the inactivation of the second allele in susceptible tissues.

The discovery of the genes in which mutations caused HNPCC was a surprise, because these were involved in the repair of mismatches in DNA, so this cancer susceptibility syndrome was due to a specific inherited DNA repair defect rather than in a faulty tumour suppressor gene. ${ }^{8}$ Somatic inactivation of the second allele led to microsatellite instability, detectable in the tumours of affected individuals, and an increased mutation rate in certain genes such as the transforming growth factor (TGF)beta receptor gene in susceptible tissues, probably responsible for the observed accelerated adenoma-to-carcinoma progression in HNPCC. It was important to understand this for clinical reasons ${ }^{9}$ because colonoscopic surveillance in such individuals needs to be more frequent (1-2-yearly) than deemed necessary in individuals without HNPCC.

During the 1990s the BRCA1 and BRCA2 breast/ovarian cancer susceptibility genes were located and identified. ${ }^{10-13}$ Germline mutations in these genes cause a high risk of developing breast cancer over a lifetime (up to $80 \%$ lifetime risk) and an increased risk of ovarian cancer (40\% in BRCA1 and 20\% in BRCA2 mutation carriers). It was found that such mutations were responsible for about $15 \%$ of familial breast cancer and a large proportion of strongly inherited cases. The analysis of pedigrees to determine the chance of finding a mutation in one of these two genes became routine in cancer genetics clinics, and with it protocols for genetic testing were developed. ${ }^{14}$ Genetic testing can be fraught with uncertainties; for instance, a gene may only have been partially screened for mutations using currently available techniques, so that a negative mutation screen result can still leave the possibility of an undetected mutation being present. Alternatively, a novel alteration in the gene might be detected, whose pathogenicity is uncertain; such results are difficult to explain, and may be misunderstood. ${ }^{15}$ However, there were enormous benefits to be derived from identifying individuals carrying such pathogenic mutations because it allowed carriers to be recognised and offered increased surveillance and prophylactic measures to reduce their cancer risks, and released at-risk individuals testing negative for the familial mutation from the need for such interventions in general.

During the 1990s our understanding of the management of individuals with these genetic conditions was revolutionised, and cancer genetics clinics were set up in all regional genetics centres, where individuals with a family history of cancer could be seen, their risks assessed, appropriate family management organised, and genetic testing offered, if appropriate. Referrals for a family history of cancer rapidly became the most common single reason for referrals to clinical genetics centres, accounting for about $40 \%$ of all referrals to the centres by 2000 . There was also a huge increase in the public awareness of this subject. The UK Cancer Genetics Group was set up for discussion of research and management issues, promoting the exchange of ideas and enabling the development of UK-wide management guidelines. There were similar developments elsewhere in Europe, and I was involved in a European BIOMED II project evaluating genetics services for breast cancer susceptibility in Europe in the late 1990s. ${ }^{16,17}$ Clinical genetics developed a new remit - that of helping to develop strategies to identify individuals at increased cancer risk in the population, eg by initiating cancer genetics clinics beyond the genetics centres, and developing agreed and audited surveillance protocols to reduce cancer morbidity and mortality in susceptible individuals. ${ }^{2,18}$ This required dialogue with health service purchasers and providers, cancer physicians, radiologists and health professionals from many other disciplines. It also became important to educate health professionals and establish strategies to identify families at moderate or high risk of inherited cancer susceptibility in the general population. In order to help develop such a system, Macmillan-funded Department of Health grants were made available in the UK to develop and evaluate models of cancer genetics service provision. As part of this enterprise, and working jointly with Ros Eeles at the Royal Marsden Hospital Foundation Trust, we compared nurse-led cancer genetics clinics in primary and secondary care in the South West Thames region with a telephone clinic service at the Royal Marsden. These models acknowledge the importance of the role of genetic counsellors and nurses in delivering such a service. Of interest in this context is the observation in our survey of cancer genetics services in European countries, that those countries which did not recognise genetic nurses and counsellors as qualified to contribute to the delivery of such services were less advanced in their service delivery than those that did. ${ }^{19}$ In many countries the role of such professionals is becoming increasingly recognised, and training structures are being put in place to ensure that appropriate competencies are achieved.

There have been startling and exciting advances in our understanding of the molecular aspects of inherited cancer susceptibility and carcinogenesis over the last 20 years, making this field a fascinating area in which to work. The concept of DNA repair 
defects as underlying many forms of cancer susceptibility has led to the discovery of lower penetrance breast cancer susceptibility genes which act in the same DNA repair pathways as BRCA1 and BRCA2, and also to the development of cancer therapy with inhibitors of other DNA repair enzymes which, in BRCA1/2 mutation carriers, appear to be more effective than in DNA repair-competent individuals. The concept of tailoring cancer therapy to the genetic background of the affected 'host' is becoming a reality. Other exciting developments include huge advances in our understanding of the importance of genomic instability and epigenetic changes including gene methylation in cancer pathogenesis. The discovery of multiple low-penetrance single nucleotide polymorphisms which can contribute to cancer susceptibility has been intriguing and is still evolving. Clearly, this rapidly developing field of science has great potential via translational research to benefit patient care, and it has been a privilege to participate in this exciting explosion of knowledge.

\section{References}

1 Lovett E. Family studies in cancer of the colon and rectum. Br J Surg 1976;63:13-8.

2 Bonelli I, Martines H, Canio M et al. Family history of colorectal cancer as a risk factor for benign and malignant tumours of the large bowel: A case-control study. Int J Cancer 1988;41:513-7.

3 Houlston RS, Murday V, Harocopos C, Williams CB, Slack J. Screening and genetic counselling for relatives of patients with colorectal cancer in a family cancer clinic. BMJ 1990;301:366-8.

4 Lynch HT, Lynch JF. 25 years of HNPCC. Anticancer Res 1994;14: 1617-24.

5 Lockhart-Mummery JP. Cancer and heredity. Lancet 1925;i:427-9.

6 Bussey HJR. Familial polyposis coli. Family studies, histopathology, differential diagnosis and results of treatment. Baltimore: Johns Hopkins University Press, 1975.

7 Groden J, Thilveris A, Samowitz W et al. Identification and characterisation of the familial adenomatous polyposis coli gene. Cell 1992;66:589-601.

8 Liu B, Parsons R, Papodopoulos N et al. Analysis of mismatch repair genes in hereditary non-polyposis colorectal cancer patients. Nat Med 1996;2:169-74.

9 Beck NE, Tomlinson IPM, Homfray T et al. Genetic testing is important in families with a history suggestive of hereditary nonpolyposis colorectal cancer even if the Amsterdam criteria are not fulfilled. Br J Surg 1997;84:233-7.

10 Hall JM, Lee MK, Newman B et al. Linkage of early onset familial breast cancer to chromosome 17q21. Science 1990;250:1684-9.

11 Miki Y, Swensen J, Shattuck-Eidens D et al. A strong candidate for the breast and ovarian cancer susceptibility gene BRCA1. Science 1994; 266:66-71.

12 Wooster R, Neuhausen SL, Mangion J et al. Localisation of a breast cancer susceptibility gene BRCA2 to chromosome 13q12-13. Science 1994;265:2088-90.

13 Wooster R, Bignell G, Lancaster J et al. Identification of the breast cancer susceptibility gene BRCA2. Nature 1995;378:789-92.

14 Hodgson SV, Morrison PJ, Irving M. Breast cancer genetics: Unsolved questions and Open Perspectives in an expanding clinical practice. Am J Med Genet 2004;129C:56-64.

15 Bish A, Sutton S, Jacobs C et al. Changes in psychological distress after cancer genetic counselling: a comparison of affected and unaffected women. Br J Cancer 2002;86:43-50.

16 Vasen HFA, Haites NE, Evans DGR et al. Current policies for surveillance and management in women at risk of breast and ovarian cancer: a survey among 16 European Family Cancer Clinics. Eur J Cancer 1998;34:1922-6.

17 Stoppa-Lyonnet D, Caligo M, Eccles D et al. Genetic testing for breast cancer predisposition in 1999; which molecular strategy and which family criteria? Dis Markers 1999;15:67-8.

18 Murday V, Pears R, Ball J et al. An audit of screening for familial breast cancer before 50 years in the South Thames Region - have we got it right? Familial Cancer 2004;3:29-34.

19 Hodgson SV, Haites NE, Caligo M et al. A survey of the current clinical facilities for the management of familial cancer in Europe. European Union BIOMED II Demonstration Project: Familial Breast Cancer: audit of a new development in medical practice in European centres. J Med Genet 2000;37:605-7.

\section{CURRENT KEY DEVELOPMENTS}

\section{Testing and screening for chromosome abnormalities}

\author{
Malcolm A Ferguson-Smith MB ChB FRCPath FRCP(Glas) FRS \\ Emeritus Professor of Pathology, Department of Veterinary \\ Medicine, Cambridge University
}

Email: maf12@cam.ac.uk

The 1959 report by Lejeune that Down's syndrome is caused by an extra 21st chromosome elicited great surprise as it was widely believed that such a gross addition of DNA could not be compatible with viability. Down syndrome was then, as now, one of the most common known causes of severe handicap, and the ability to observe the extra chromosome by ordinary microscopy was responsible for the rapid emergence of clinical cytogenetics. The sex chromosome abnormalities and other less common trisomies soon followed the discovery of trisomy 21 , and, later, other abnormalities, due to loss or gain of parts of chromosomes, were recognised. Over the years, new techniques have been developed to identify progressively smaller chromosome defects, each characterised by specific and non-specific malformations. The most modern techniques do not require microscopy as they exploit the DNA sequence of the human genome, using molecular methods easily capable of a resolution of several thousand DNA base pairs. The difficulty nowadays is to distinguish individual DNA variation of no clinical significance from changes which lead to severe pathology. The distinction usually requires the testing of the patient's parents.

Up to 1975, the usual indication for prenatal diagnosis of Down's syndrome was a maternal age of 35 years or over. It is still not known why increased maternal age raises the risk, but only 